\title{
Augmented Reality for Educational Enhancement
}

\author{
Abdullah Mubarak Ghare ${ }^{1}$, Mohammed Akhtar Khan ${ }^{1}$, Mustafa Rangwala ${ }^{1}$, Shadab Kazi ${ }^{1}$, \\ Prof. Sameer Panwala ${ }^{1}$, Prof. Abdus Salam ${ }^{1}$ \\ Department of Computer Engineering, Anjuman-I-Islam Kalsekar Technical School of Engineering, University of \\ Mumbai, Mumbai, India ${ }^{1}$
}

\begin{abstract}
Augmented Reality superimposes virtual objects over real world environment. Educators know that learning deepens, not just through reading and listening, but also through creating and interacting. This proposed project of ours aims to enhance current educational system using Augmented Reality. We are developing an application which will show animations, videos and 3D models of educational material. This project can enhance learning, creativity and retention among students. Our application will also foster intellectual curiosity among students making them smarter. We aim to change the conventional way of education and open a new opportunity towards a smart classroom.
\end{abstract}

Keywords: Augmented Reality; education; children; learning; virtual; disabled children; interaction; android.

\section{INTRODUCTION}

As computing environment changes, new interfaces have been introduced to provide natural interaction between human and computers [1]. Augmented Reality (AR) is a technology that allows computer-generated virtual imagery information to be overlaid onto a live direct or indirect real-world environment in real time [2]. For educators and designers, defining AR in a broad sense would be more productive because such a definition suggests that AR could be created and implemented by varied technologies, such as desktop computers, handheld devices, head-mounted displays and so on [3]. In AR, the environment is real life feed, but it is extended with some information and image from the system. AR takes advantage of virtual objects or information overlaying physical objects or environments, resulting in a mixed reality in which virtual objects and real environments coexist in a meaningful way to augment learning experience [4]. Augmented Reality and Virtual Reality use same hardware technologies and share lots of factors like computer generated virtual scenes, 3D objects and interactivity. The main difference between them is where virtual reality aims to replace the real world while augmented reality respectfully supplements it. AR allows the user to be immersed in the created environment by mixing the rendered media with the real environment.

It may seem to be little surprising but statistical reports show that by 2020, around 70 percent of the world population will own a smart phone. We believe that we should be embracing these opportunities. For instance, we see AR as a real opportunity for colleges and universities as a way to communicate with learners and improve the learning experience. The fundamental requirements of $A R$ application include a camera, a screen display, a memory and a processor to render virtual components. Various numbers of educational possibilities can be provided to students using AR technology which will help students increase their interest in the presented material and increase their learning outcome [5]. Augmented reality helps teachers to add digital contents with lot of information as well as geographic locations about a place or object. Digital information appears on the screen when you scan any object or place using your tablet, phone or smart devices with AR technology. This digital information is gathered from 3D models, various website, video, etc. Due to advancement in mobile technology, it is now feasible to use Augmented Reality (AR) technology for learning. There have been some attempts to use AR for training and educational purpose. The recent development in mobile devices makes it possible for mobile AR environments to support outdoor learning enhanced by computer simulations and virtual objects with the focus on real environments.

The physical and interpersonal interaction is intended to permanently bind the experience to the visitor's mind. The purpose of free learning Education experience is to inspire curiosity, create a positive attitude towards the topic and engage the visitor in a memorable experience that inspires discussion long it is encountered.[6]

AR is being used in an increasing variety of ways, from providing point-of-sale information to shoppers, tourist information on landmarks, computer enhancement of traditional printed media, service information for on-site engineers; the number of applications is huge.

The technologies that make AR possible are much more powerful than ever before and compact enough to deliver AR experiences to not only corporate settings but also academic venues through personal computers and mobile devices, several educational approaches with AR technology are more feasible.[7]

Traditionally, the visuals available to students in the books are two dimensional pictures which may be very much confusing. The main problems with traditional learning methods are maintaining the interest of the students into the learning process. Now, AR can attract learners from a 
number of different perspectives that have not previously been taken into consideration, Augmented Reality for Educational Enhancement is believed to be better than ever before due to improvements in Computer and Information Technology. AR for Education helps easier understanding of subject. It increases curiosity and motivation to learn amongst students. Higher level of concentration and memory retention is observed amongst students who can visualize what they learn. Hence, it results in increased satisfaction in the content that they have learnt. AR systems provide solutions for learning difficulties that have been identified in previous research. For example, students usually encounter difficulties visualizing unobservable phenomena such as spinning of the earth [8]. This suggests that, AR provides new possibilities and challenges in education.

AR can be applied in different fields of education ranging from learning alphabets in primary schools to studying different body parts in post-graduate studies. Using AR we can visualize places which can't be explored in real life. Learning mathematical formulas after visualizing how it is stated becomes very easy for students.

Special children require special education. Children with learning disabilities, communication disorders, emotional and behavioural disorders, physical disabilities and developmental disabilities can be benefitted by the use of AR Technology. It breaks the barrier of language as an image can be understood by everyone. Deaf students are also benefitted as deaf people do not have a specific sign language to communicate with others. They don't like being interpreted again and again. By AR, deaf students can learn at their own pace without any problems. It can be used in various other fields of education for benefitting everyone.

Another study conducted by researchers from Vienna University of Technology [9] aims to study the benefits of using AR applications in mathematics. The study states that traditionally students will struggle in visualizing and dealing with three dimensional objects using pen and paper. Using AR applications the students will be able to see directly the three dimensional objects. In this paper, we explore the use of augmented reality for education purpose.

\section{LITERATURE REVIEW}

Multiple research papers have illustrated the integration of AR technology with education and possible beneficial results that can be derived from this new technological evolution. All papers that we referenced show that AR enhances the quality of education.

Many AR applications already exist like The Magic Book, The DehAR Book, The SUN-RA, GeoAR, and The Book of Augmented Chemical Reactions. The Magic Book is actually a normal book that can be read in the traditional way; however, the user has the option to see it behind a display that would show 3D models so as to greatly enrich its content [5]. The Magic Book aimed to teach 9-year old students how to mix primary colors to get new colors and the application was found to be very effective. DehAR book aims to enhance engineering student's spatial skills and was tested on freshmen mechanical engineering students. This AR application displayed 3D models to show the components needed to perform spatial-ability related tasks.

The SUNRA is an AR application that aims to enhance student's understanding about the solar system. This application shows live videos of the solar system as well as different audio clips related to different planet animations. Another remarkable work was the GeoAR which is an AR application for teaching geometry. The GeoAR book shows a marker in every page as well as some explanation on different geometric shapes like rectangles, circles, triangle, etc. This application was tested in a classroom and had yielded positive results. The application was concluded to be a very attractive as well as simulative because of the AR-based animations, audios, and $3 \mathrm{D}$ representation of the objects.

Paper [10] described about their application SMART (System of augmented reality for teaching) that is an educational system using AR technology. There project uses AR technology for teaching low education level concepts, such as the means of transportation and types of animals. There system overlays virtual models such as truck, car and airplane, on the real time video feed shown to whole class.

Mobile Solution for Brain Visualization Using Augmented and Virtual Reality, in this paper they described solution for visualizing the brain using Augmented Reality and Virtual Reality techniques. Drawback in this paper is the stability of the alignment of the virtual models.

One type of AR technologies includes a head-mounted display and/or additional backpack with computer equipment. The cumbersome and expensive design could cause problems such as discomfort and poor depth perception [8]. Another issue is regarding a trade-off in technology design between location dependency and independency (Klopfer \& Sheldon, 2010). While locationspecific technologies contextualize student's learning, provide a connection to a particular location, and help students give new meaning to their familiar locations, location-independent design has advantages in portability and flexibility that does not require teachers and students to be present in specific locations and could save great cost on transportation.

Like many educational innovations in the past, the use of AR in classrooms could encounter constraints from schools and resistances among teachers. The nature of usual AR system approaches however is quite different from the teacher-centered, delivery-based focus in conventional teaching methods [8][11]. AR has not been much adopted into academic settings due to little financial support from the government and lack of the awareness of needs for AR in academic settings.[12]. There are a great number of studies going on to improve the compatibility and applicability of AR into real life. However, according 
to [13], many questions still linger about its use in education and training, including issues of cost effectiveness, of efficiency between AR instructional systems and conventional methods, and the like.

According to the Research, the average score of the student's perception of mobile AR activity is 7.6 which is above the mean score of student's perceptions which is 7.3. Once student indicated "Using the tablet computer to go outside and actually collect data allows me to get a sense of the unit quickly." Overall the student's perceptions of this AR activity were positive. In general, the students made significant improvements on the 15 conceptual items from the pretest to the posttest. This result indicates that an SSI unit enhanced by mobile AR can improve student's learning of the science concepts.[14]

AR will make educational environments more productive, pleasurable, and interactive than ever before. AR can engage a student in various interactive ways that have never been possible before and provide each individual with one's unique discovery path with highly rich content from computer-generated 3D environments and models.

Many researches have come to recognize that learning through play, and teacher guidance can help children to get over their initial fears and even begin to enjoy these subjects. Many paper referred states that, Augmented Reality based books and Applications have been made for educational purposes but it is restricted to self study and are not being used by institutes. One of the main drawbacks in all papers is that we are unable to interact with the object.

\section{PROBLEM STATEMENT}

Education to a child is like a sword is for a warrior. Current educational patterns in most countries do not induce much interest amongst the student. Above this, availability of education for uniquely gifted children is even rare. Forming interest in education for one and all still remains to be one of the major concerns in educating children.

We propose a solution to this dying interest in learning. Giving life to textbook contents can have a major impact on their interest. We plan to bring objects at a distance of hand which were once unimaginable. Seeing the objects interact to gestures and seeing them at such close distance can help children remember content for a very long time.

[15] Billinghurst determined that people, especially young children, can read books in more interactive and realistic ways by superimposing 3D rendered models onto books with AR technology.

Knowledge is the only existing superpower. There is too much to learn but there are not many to teach. With AR in education, knowledge will be available to whoever desires to learn and that too at the blink of an eye.
IV. PROPOSED MODEL

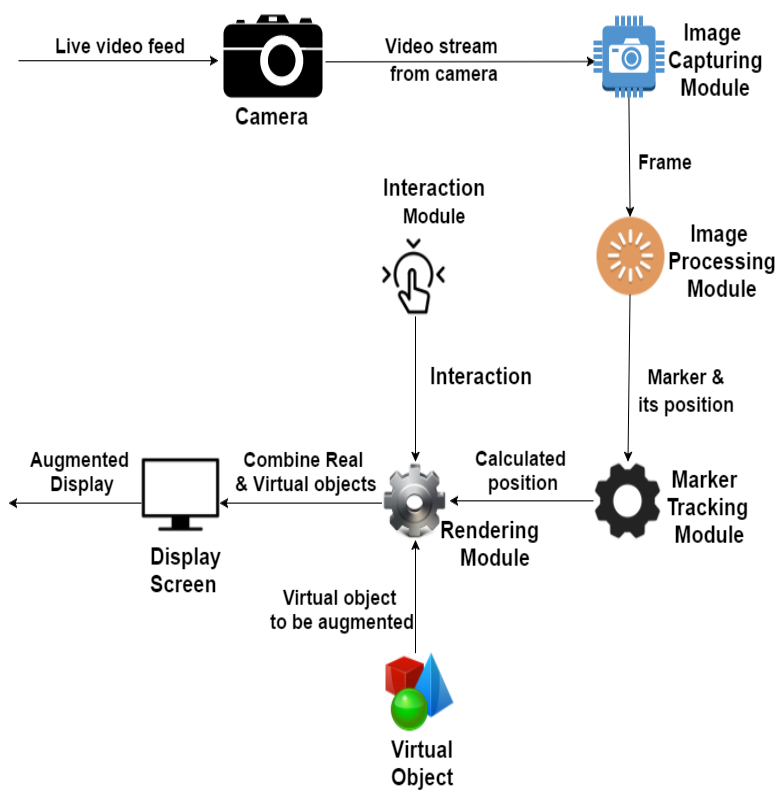

fig: Proposed System Architecture

We just require a smart phone. The inbuilt camera perceives live feed from the environment and sends it to the image capturing module where it separates the live feed into frames and one that frame is sent to image processing module. Here the image processing module detects the marker and sends it position to marker tracking module. Now the marker is identified and it is sent to the rendering module. In the rendering module, a virtual object is matched to the marker and that virtual object is superimposed on the real video scene. Finally, the augmented video is displayed on the screen. Now whenever the user interacts with the virtual objects then accordingly the response is generated and response is shown on the screen.

We are introducing various methods for interaction with virtual objects which enable user to understand the study materials more clearly. Types of interaction include touch based, gesture based and voice based interactions. We plan to use recent and faster image processing algorithm for quicker marker detection. We will use high graphic processors and a high resolution camera for lesser chances of disturbances and faster image rendering. We will make images that have high contrasting colours. We plan to create highly detailed 3D model or animation, to avoid distortions in augmented feed.

\section{V.CONCLUSION}

Augmented Reality has a bright future as it promises better interaction with real and virtual world in ways which has previously been unimaginable. It is a new way of interaction with User Interface. Augmented Reality is yet to mature and has endless possibilities to explore. We plan to make informative augmentable content which matches the teaching pattern and syllabus of institutes. In this way, 
our augmentable books can be used by institutes for teaching their syllabus in an easier way. We plan to use this technology for the benefit of children with learning disabilities as visualizations are very helpful for them. We propose to add a module which allows interaction with augmented objects. With Augmented Reality in Education, "LEARNING WILL NEVER BE A BARRIER FOR ANYONE".

\section{REFERENCES}

[1] C. Elliot, G. Schechter, R. Yeung, and S. Abi-Ezzi: "TBAG: A High Level Framework for Interacive, Animated 3D Graphics Applications," proceedings of ACM SiGGRAPH 9 4, pp.421-434 (1994)

[2] Azuma, 1997; Zhou, Duh, \& Billinghurst, 2008

[3] Broll et al., 2008; Johnson et al., 2010b; Liu, 2009

[4] Arvinitis et al, 2007; Dunleavy, Dede \& Mitchell, 2008

[5] AIR-EDUTECH, Lamees Mahmoud Mohd Said Al Qassem, Hessa Al Hawai, M. Jamal Zemerly, 2016

[6] Charles E. Hughes, Christopher B. Stapleton, Darin E. Hughes, and Eileen M. Smith "Mixed Reality in Education, Entertainment, and Training", 2005

[7] Kangdon Lee, "Augmented Reality in Education and Training", 2012

[8] Kerawalla et al., 2006

[9] M. Billinghurst, "Augmented Reality in Education", New horizons for learning IX, October 2003

[10] Mitchell, 2011; Squire \& Jan, 2007

[11] M. Billinghurst, “Augmented Reality in Education”, New horizons for learning IX, October 2003

[12] Hsin-Yi Chang, Hsin-Kai Wu and Ying-Shao Hsu; "Integrating a mobile augmented reality activity to contextualize student learning of a socioscientific issue, 2013

[13] Kangdon Lee, "Augmented Reality in Education and Training", 2012

[14] Freitas, R., \& Campos, P. (2008). SMART: a System of augmented reality for teaching 2nd grade students. Proceedings of the 22nd British Computer Society Conference on Human-Computer Interaction (HCI 2008), 27-30. Liverpool John Moores University, UK

[15] M. Billinghurst, H. Kato and I. Poupyrev, "The Magicbook: Moving Seamlessly Between Reality and Virtuality", IEEE Computer Graphics and Applications, vol. 21, no. 3, pp. 6-8, May/June 2001. 